\title{
Total Syntheses of Tambjamines C, E, F, G, H, I and J, BE-18591, and a Related Alkaloid from the Marine Bacterium Pseudoalteromonas tunicata
}

2007

Vol. 9, No. 24

$5127-5130$

\author{
David M. Pinkerton, Martin G. Banwell, ${ }^{*}$ and Anthony C. Willis
}

Research School of Chemistry, Institute of Advanced Studies, The Australian National University, Canberra, ACT 0200, Australia

mgb@rsc.anu.edu.au

Received October 4, 2007

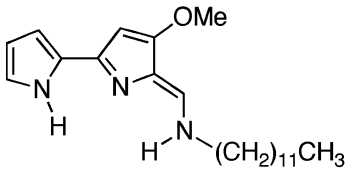

BE-18591 (5)<smiles>C/C=C\CCN/C=C1\N=C(c2ccc[nH]2)C=C1OC</smiles>

"new" Tambjamine (6)<smiles>CCCCCCNC=C1N=C(c2ccc(Br)[nH]2)C=C1OC</smiles>

Tambjamine J (11)

The acetate salts of tambjamines C, E, and F (2-4, respectively), as well as those of the related alkaloids BE-18591 (5) and 6, have been prepared by treatment of bipyrrole aldehyde 16 with the relevant amine in the presence of acetic acid. The $5^{\prime}$-bromo-analogue, 30, of compound 16 has also been prepared and used to obtain the acetate salts of tambjamines $G, H, I$, and $J$ (8-11 respectively).

The tambjamines $(\mathbf{1}-\mathbf{1 2})^{1}$ are a group of bipyrrolic alkaloids that have been isolated from various marine and terrestrial sources. ${ }^{2}$ In some instances they have been implicated in the chemical defense mechanisms of the organisms from which they were first obtained. ${ }^{3}$ A number of these compounds have also been shown to possess intriguing biological

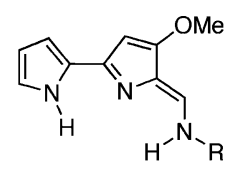

$1 \mathrm{R}=\mathrm{H}(\mathrm{T} . \mathrm{A})$

$2 \mathrm{R}=\mathrm{CH}_{2} \mathrm{CH}\left(\mathrm{CH}_{3}\right)_{2}$ (T.C)

$3 \mathrm{R}=\mathrm{CH}_{2} \mathrm{CH}_{3}$ (T.E)

$4 \mathrm{R}=\left(\mathrm{CH}_{2}\right)_{2} \mathrm{Ph}$ (T.F)

$5 \mathrm{R}=\left(\mathrm{CH}_{2}\right)_{11} \mathrm{CH}_{3}(\mathrm{BE}-18591)$

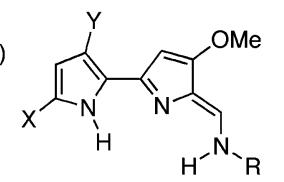

$7 \mathrm{R}=\mathrm{H}, \mathrm{X}=\mathrm{Br}, \mathrm{Y}=\mathrm{H}$ (T.B)

$8 \mathrm{R}=\mathrm{CH}_{2} \mathrm{CH}_{3}, \mathrm{X}=\mathrm{Br}, \mathrm{Y}=\mathrm{H}$ (T.G)

$9 \mathrm{R}=\mathrm{CH}_{2} \mathrm{CH}_{2} \mathrm{CH}_{3}, \mathrm{X}=\mathrm{Br}, \mathrm{Y}=\mathrm{H}(\mathrm{T} . \mathrm{H})$

$10 \mathrm{R}=\mathrm{CH}_{2} \mathrm{CH}\left(\mathrm{CH}_{3}\right)_{2}, \mathrm{X}=\mathrm{Br}, \mathrm{Y}=\mathrm{H}(\mathrm{T} . \mathrm{I})$

$11 \mathrm{R}=\mathrm{CH}_{2} \mathrm{CH}\left(\mathrm{CH}_{3}\right) \mathrm{CH}_{2} \mathrm{CH}_{3}, \mathrm{X}=\mathrm{Br}, \mathrm{Y}=\mathrm{H}$ (T.J)

$12 \mathrm{R}=\mathrm{CH}_{2} \mathrm{CH}\left(\mathrm{CH}_{3}\right)_{2}, \mathrm{X}=\mathrm{H}, \mathrm{Y}=\mathrm{Br}$ (T.D) properties, a feature that is probably unsurprising given their structural relationship to the tripyrrolic prodigiosin family of alkaloids, members of which display very promising cytotoxic and immunosuppressive properties. ${ }^{4}$ For example, tambjamine 5 (aka BE-18591) has been shown to inhibit immunoproliferation and gastritis in rabbits, ${ }^{5}$ while screening of tambjamine I (10) against a 60-cell-line panel shows that

(1) For convenience, and because they each possess the same bipyrrolic core, we have designated all of the natural products 1-12 as tambjamines even though one of these, $\mathbf{5}$, has been assigned the code-name BE-18591 while another, 12, has only been identified as a "...new member of the tambjamine class..." and remains to be given a letter designation.

(2) (a) Carté, B.; Faulkner, D. J. J. Org. Chem. 1983, 48, 2314 (tambjamines A-D). (b) Lindquist, N.; Fenical, W. Experientia 1991, 47, 504 (tambjamines E and F). (c) Kojiri, K.; Nakajima, S.; Suzuki, H.; Okura, A.; Suda, H. J. Antibiot. 1993, 46, 1799 (BE-18591). (d) Blackman, A. J.; Li, C. Aust. J. Chem. 1994, 47, 1625 (tambjamines G-J). (e) Franks, A.; Haywood, P.; Holmström, C.; Egan, S.; Kjelleberg, S.; Kumar, N. Molecules 2005, 10, 1286 (cmpd 6).

(3) Carté, B.; Faulkner, D. J. J. Chem. Ecol. 1986, 12, 795.

(4) For reviews of such topics see: (a) Manderville, R. A. Curr. Med. Chem. - Anti-Cancer Agents 2001, 1, 195. (b) Fürstner, A. Angew. Chem. Int. Ed. 2003, 42, 3582. See also: (c) Baldino, C. M.; Parr, J.; Wilson, C. J.; Ng, S.-C.; Yohannes, D.; Wasserman, H. H. Bioorg. Med. Chem. Lett. 2006, 16, 701 . 
it has average $\mathrm{GI}_{50}$ and $\mathrm{LC}_{50}$ values of 1.6 and $18 \mu \mathrm{M}$, respectively. ${ }^{4 a}$ Certain tambjamines have also been observed to bind duplex DNA and can cleave this biomolecule in the presence of $\mathrm{Cu}(\mathrm{II}){ }^{4 a}$ While the tambjamines have been manipulated chemically for the purposes of securing a library of analogues, ${ }^{6}$ they do not appear to have been the subject of any total synthesis studies. Accordingly, we now report the de novo preparation of all but three members of the class using straightforward and unambiguous methods that have permitted the confirmation of the structures of these compounds. The present work should also allow for a more comprehensive evaluation of the biological properties of these intriguing alkaloids.

The route used in establishing total syntheses of tambjamines $\mathbf{2}-\mathbf{5}$ is shown in Scheme 1. This relies upon the

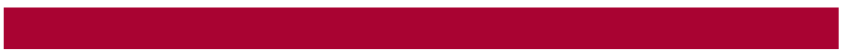

Scheme 1

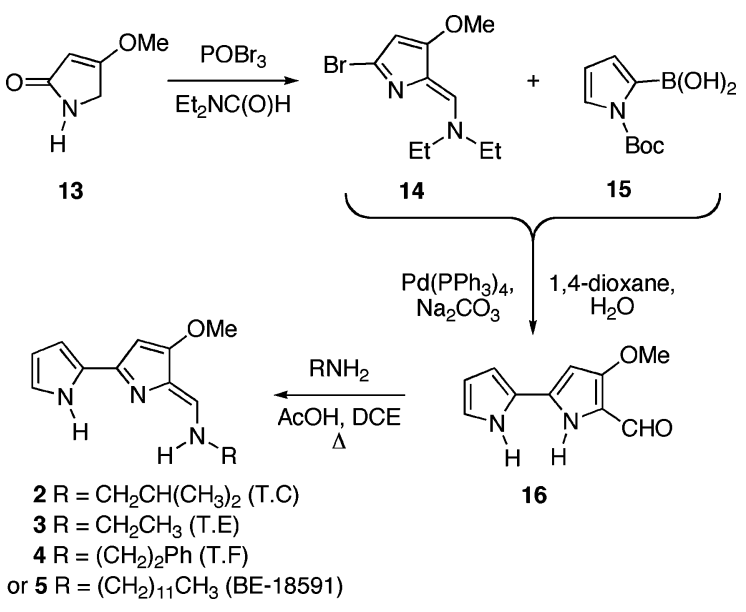

recently reported and very concise protocol of Lavallée and co-workers ${ }^{7}$ for the synthesis of aldehyde $\mathbf{1 6}$, a pivotal but previously difficult to access intermediate associated with various syntheses of prodigiosin ${ }^{4}$ and a compound that is also generated by base hydrolysis of the non-brominated tambjamines. ${ }^{6}$ Thus, as reported by Lavallée and co-workers, ${ }^{7}$ commercially available 4-methoxy-3-pyrolin-2-one (13) was subjected to a Vilsmeier-Haack reaction using $\mathrm{POBr}_{3}$ and diethylformamide, and the ensuing azafulvene 14 (49\%) engaged in a Suzuki-Miyaura cross-coupling reaction ${ }^{8}$ with the readily available boronic acid $15^{9}$ to give the required aldehyde $\mathbf{1 6}$ in $95 \%$ yield. Reaction of a solution of this last compound in 1,2-dichloroethane (DCE) with the relevant range of commercially available alkyl amines in the presence

(5) Tanigaki, K.; Sato, T.; Tanaka, Y.; Ochi, T.; Nishikawa, A.; Nagai, K.; Kawashima, H.; Ohkuma, S. FEBS Lett. 2002, 524, 37.

(6) Davis, R. A.; Carroll, A. R.; Quinn, R. J. Aust. J. Chem. 2001, 54, 355.

(7) Dairi, K.; Tripathy, S.; Attardo, G.; Lavallée, J.-F. Tetrahedron Lett. 2006, 47, 2605.

(8) For a review of the palladium-catalyzed cross-coupling and related reactions involving pyrroles see: Banwell, M. G.; Goodwin, T. E.; Ng, S.; Smith, J. A.; Wong, D. J. Eur. J. Org. Chem. 2006, 3043.

(9) Martina, S.; Enkelmann, V.; Wegner, G.; Schlüter, A.-D. Synthesis 1991, 613 of acetic acid at $18-50{ }^{\circ} \mathrm{C}$ then afforded the acetate salts of tambjamines $\mathbf{2}-\mathbf{5}$ in yields ranging from 69 to $100 \%$. For example, treatment of aldehyde $\mathbf{1 6}$ with 1-aminododecane under the specified conditions produced the acetate salt of BE-18591 (5) in 100\% yield. The spectral data derived from this material matched those reported for the natural product. ${ }^{2 c, 10}$ Thus far, we have been unable to identify conditions under which aldehyde $\mathbf{1 6}$ reacts with ammonia or a surrogate thereof so as to provide useful quantities of tambjamine A (1).

The unsaturated primary amine required for the preparation of tambjamine $\mathbf{6}$ by the method just described has not been reported in the literature but was readily generated by the route outlined in Scheme 2. Thus, following a protocol

\section{Scheme 2}

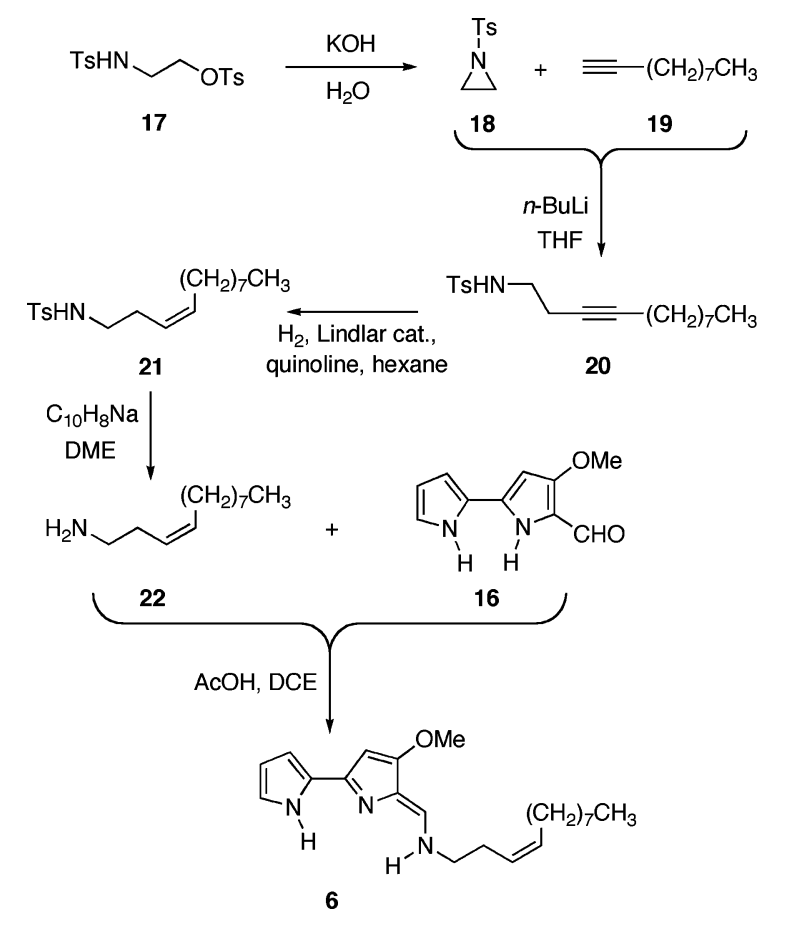

reported by Bulkowski, ${ }^{11}$ the bis-tosyl derivative, 17, of ethanolamine was treated with aqueous $\mathrm{KOH}$ to give aziridine $\mathbf{1 8}^{11}$ (96\%) that was then subjected to nucleophilic ring-opening with the anion derived from 1-decyne (19) using a procedure reported by Gronquist and Meinwald. ${ }^{12}$ The ensuing internal alkyne 20 (42\%) was subject to hydrogenation using Lindlar's catalyst in the presence of quinoline ${ }^{13}$ so as to afford the $Z$-alkene $\mathbf{2 1}$ in $\mathbf{9 9 \%}$ yield. Reductive cleavage of the sulfonamide residue within this last compound using sodium naphthalenide in 1,2-dimethoxyethane

(10) We believe such comparisons are legitimate since it has been reported (see ref $2 \mathrm{~d}$ ) that there is little difference between the NMR spectra of various tambjamines and those of the corresponding acetate salts.

(11) Martin, A. E.; Ford, T. M.; Bulkowski, J. E. J. Org. Chem. 1982, 47, 412.

(12) Gronquist, M. R.; Meinwald, J. J. Org. Chem. 2001, 66, 1075.

(13) Overman, L. E.; Brown, M. J.; McCann, S. F. Org. Synth. 1989, $68,182$. 
Scheme 3

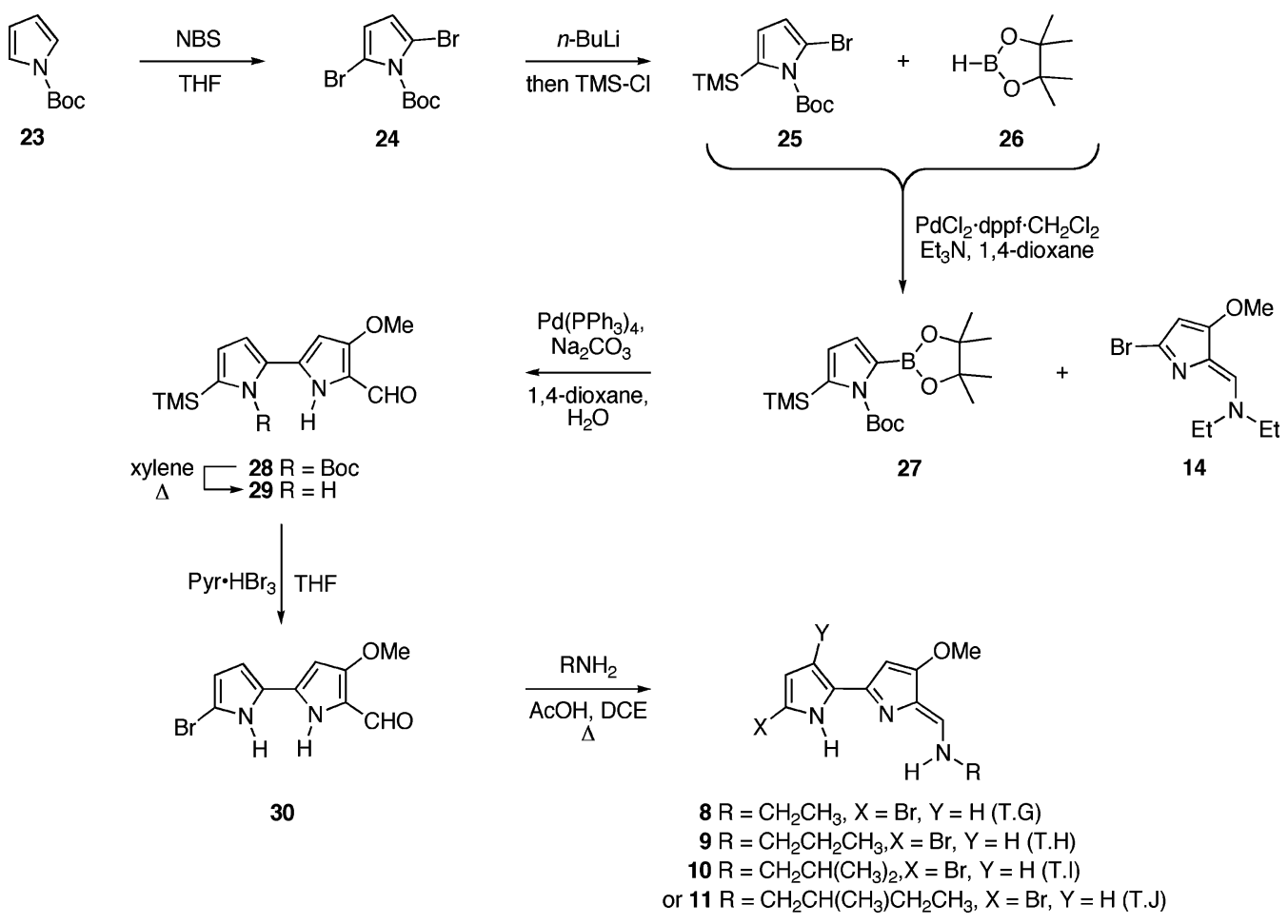

$(\mathrm{DME})^{14}$ then afforded the required amine $22(72 \%)$ that engaged in the by now standard condensation with aldehyde 16 to give tambjamine $6(32 \%)$. The ${ }^{1} \mathrm{H}$ and ${ }^{13} \mathrm{C}$ NMR spectra derived from compound $\mathbf{6}$ match those recorded on the natural product. ${ }^{2 \mathrm{e}}$ Furthermore, the infrared and mass spectral data obtained on the synthetic material were in full accord with the assigned structure.

Our initial attempts to generate the requisite bromoanalogues of compound $\mathbf{1 6}$ that could be used to prepare tambjamines 7-12 involved treating the aldehyde $\mathbf{1 6}$ with various electrophilic brominating agents. Unfortunately, under all the conditions investigated only complex mixtures of products were obtained. Accordingly, directed syntheses of such compounds were pursued. Eventually, the route shown in Scheme 3 was established as a method for obtaining the $5^{\prime}$-bromo-analogue, 30, of compound 16. Thus, following protocols established by Weinreb et al., ${ }^{15}$ the readily available $\mathrm{N}$-Boc protected pyrrole $\mathbf{2 3}$ was subjected to 2-fold bromination with $N$-bromosuccinimide (NBS) and the ensuing 2,5dibrominated derivative $\mathbf{2 4}(98 \%)$ treated with $n$-BuLi then trimethylsilyl chloride (TMS-Cl). In this manner the $C$ silylated compound $\mathbf{2 5}{ }^{15}$ was obtained in $61 \%$ yield. Miyaura borylation of this material ${ }^{8}$ using borane $\mathbf{2 6}$ then afforded the pyrrole $27(80 \%)$ that could be engaged in a SuzukiMiyaura cross-coupling reaction with azafulvene $\mathbf{1 4}$ so as to afford a chromatographically separable mixture of com-

(14) Ji, S.; Gortler, L. B.; Waring, A.; Battisti, A.; Bank, S.; Closson, W. D.; Wriede, P. J. Am. Chem. Soc. 1967, 89, 5311.

(15) Stein, D.; Anderson, G. T.; Chase, C. E.; Koh, Y.-h.; Weinreb, S. M. J. Am. Chem. Soc. 1999, 121, 9574. pounds $28(23 \%)$ and $29(60 \%)$. Heating the former product in refluxing xylene effected thermolytic cleavage of the associated Boc-group and thus delivered further quantities of the TMS-derivative $\mathbf{2 9}(\mathbf{1 0 0 \%})$ of the aldehyde $\mathbf{3 0}$. The spectral data derived from compound $\mathbf{2 9}$ were in full accord with the assigned structure, but final confirmation of this followed from a single-crystal X-ray analysis. The derived ORTEP diagram is shown in Figure 1. Disappointingly, the silylated pyrrole 29 failed to engage in a clean ipso-

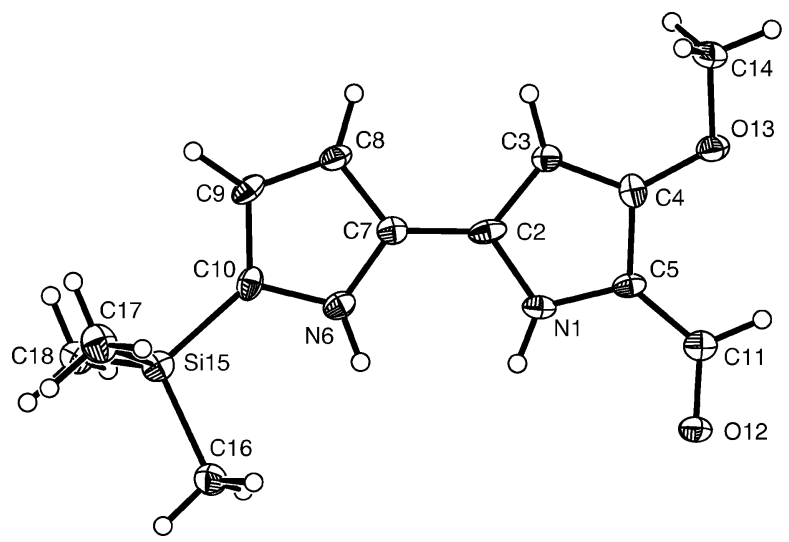

Figure 1. Structure of molecule one of $\mathrm{C}_{13} \mathrm{H}_{18} \mathrm{~N}_{2} \mathrm{O}_{2} \mathrm{Si}$ (29) with labeling of selected atoms. Anisotropic displacement ellipsoids show $30 \%$ probability levels. Hydrogen atoms are drawn as circles with small radii. 
substitution reaction upon exposure to NBS. In contrast, reaction of substrate $\mathbf{2 9}$ with freshly prepared pyridinium hydrobromide perbromide ${ }^{16}$ led to the desired product $\mathbf{3 0}$ in $76 \%$ yield. Reaction of this last compound with the relevant range of alkyl amines in the presence of acetic acid under the same sorts of conditions defined above then afforded, after conventional flash chromatographic purification over silica gel, the acetate salts of tambjamines 8-11 in yields ranging from 81 to $99 \%$. The spectral data derived from each of these materials matched those reported for the corre-

(16) Fieser, L. F.; Fieser, M. Reagents for Organic Synthesis; John Wiley and Sons: New York, 1967; p 967. sponding natural products, some of which have also been isolated as their acetate salts. ${ }^{2 \mathrm{~d}}$

Acknowledgment. We thank the Institute of Advanced Studies and the Australian Research Council for generous financial support.

Supporting Information Available: Full experimental procedures; ${ }^{1} \mathrm{H}$ and/or ${ }^{13} \mathrm{C}$ NMR spectra of compounds $\mathbf{2}-\mathbf{6}$, 8-11, 14, 16, 20-22, and 27-30; the single-crystal X-ray data for compound 29 (CCDC number 659930). This material is available free of charge via the Internet at http://pubs.acs.org.

OL7024313 